\title{
Eine große Koalition der Vernunft
}

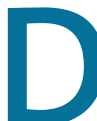

er Koalitionsvertrag ist unterschrieben, die erste Bundeskanzlerin ist gewählt. Im Bundesgesundheitsministerium wird über Wege der Finanzierbarkeit und Kosteneinsparungen von Gesundheitsleistungen gestritten. Bürgerversicherung und Ende der Zweiklassenmedizin sind die Schlagwörter, die in der Bevölkerung und in der Ärzteschaft für Unruhe sorgen. Die Sparpotenziale im ärztlichen Bereich sind erschöpft. Nach den ersten Abrechnungsergeb-

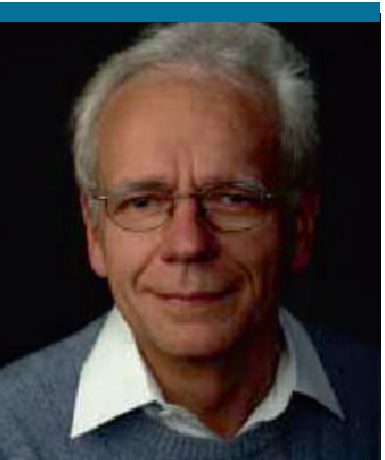

Dr. Wolfgang Rebien, Hamburg, Präsident des ÄDA

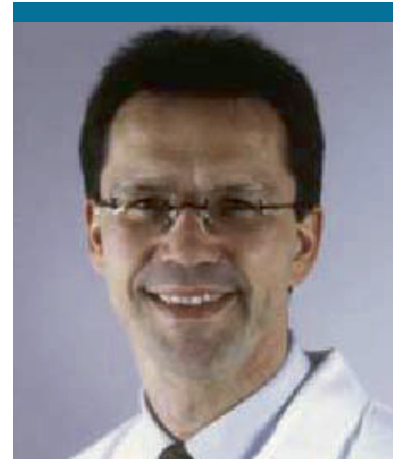

Prof. Dr. Ludger Klimek, Zentrum für Rhinologie und Allergologie, Wiesbaden, Vorstandsmitglied des ÄDA

bundes Dermatologischer Kliniken (IVDK), der mehr als 40 Allergieabteilungen in Deutschland, Österreich und der Schweiz vereint. Durch die Arbeit des IVDK werden wertvolle Informationen zur Bedeutung von Kontaktallergenen gesammelt. Die Arbeit von Pfaar et al. (S. 630) beschäftigt sich mit der historischen Entwicklung und dem heutigen Stand des Wissens zur Acetylsalicylsäure-Intoleranz am Beispiel des bedeutenden Allergologen Max Samter.

Wenn also trotz der ge2005 (EBM 2000 plus) wird im niedergelassenen Facharztbereich in Nieder-

gesellschaften herausgegebene Taschenbuch „Allergien, Volkskrankheiten des 21. Jahrhunderts" dienen. sachsen und Brandenburg über Umsatzeinbußen von bis zu 30\% berichtet.

Ein hohes Kostenpotenzial liegt auch in der Versorgung von allergiekranken Patienten. Immerhin sind 30 bis $40 \%$ der Bevölkerung an einer Allergie erkrankt. Bei dieser hohen Anzahl von Patienten bleibt es nicht aus, dass gerade im Diagnostikbereich durch ungezielte Untersuchungen und Doppeluntersuchungen Gelder des Gesundheitswesens verschwendet werden.

Die großen Fachgesellschaften der Allergologie (ÄDA, DGAKI, GPA und DAAU) haben sich in den letzten Jahren bemüht, patientengerechte und ökonomische Qualitätsrichtlinien zur Diagnose und Therapie allergischer Erkrankungen zu erstellen. Nachdem der erste Entwurf dieser Richtlinien bisher aus schwer verständlichen Gründen nicht zur Umsetzung kam, wird jetzt nochmals in Zusammenarbeit mit dem Gesundheitsministerium und den anderen gesundheitspolitischen Gremien um eine Neuauflage gerungen.

Als Basis für diese Diskussion soll insbesondere das kürzlich von den Fach-
In diesem Buch werden die wichtigsten Aussagen eines berufspolitischen Spitzengesprächs vom Dezember 2004 zu den Problemen der Versorgung allergiekranker Patienten in Deutschland zusammengefasst.

Doch glücklicherweise können sich Ärzte auch im auslaufenden Jahr 2005 noch mit ärztlichen Tätigkeiten zum Wohle der Patienten beschäftigen. Dies zeigt die $\mathrm{Pu}-$ blikation von Schnuch et al. (S. 618) über die Tätigkeit des Informationsver-

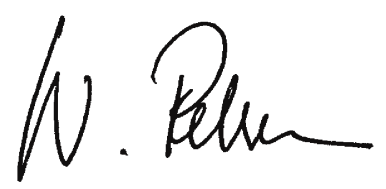

Dr. Wolfgang Rebien sundheitspolitischen Großwetterlage noch gute Medizin gemacht wird, besteht für die Zukunft Hoffnung auf eine große Koalition der Vernunft im Gesundheitswesen. In diesem Sinne möchten wir Sie auch bereits heute zum 29. Kongress des Ärzteverbandes Deutscher Allergologen vom 15. bis 17. September 2006 in die herrlichen Räumlichkeiten des Kurhauses in Wiesbaden einladen - Hauptthema: „Allergie - Volkskrankheit des 21. Jahrhunderts".

Wir wünschen Ihnen im Namen aller Herausgeber einen friedlichen und besinnlichen Jahresausklang 2005 - und für uns Allergologen ein besseres Jahr 2006 !

Ihre

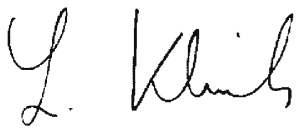

Prof. Dr. Ludger Klimek 